\title{
Syntaxin 5 Overexpression and $\beta$-Amyloid 1-42 Accumulation in Endoplasmic Reticulum of Hippocampal Cells in Rat Brain Induced by Ozone Exposure
}

\author{
Luis Fernando Hernández-Zimbrón and Selva Rivas-Arancibia \\ Departamento de Fisiología, Facultad de Medicina, Universidad Nacional Autónoma de México, 04510 México, DF, Mexico \\ Correspondence should be addressed to Selva Rivas-Arancibia; srivas@unam.mx
}

Received 19 January 2016; Revised 31 March 2016; Accepted 3 April 2016

Academic Editor: Yiying Zhang

Copyright (C) 2016 L. F. Hernández-Zimbrón and S. Rivas-Arancibia. This is an open access article distributed under the Creative Commons Attribution License, which permits unrestricted use, distribution, and reproduction in any medium, provided the original work is properly cited.

\begin{abstract}
Oxidative stress is a risk factor for Alzheimer's disease and it is currently accepted that oxidative damage precedes the overproduction of A42 peptide. We have reported that ozone causes oxidative stress inducing neurodegeneration in the brain of rats. It is associated with A42 overproduction and intracellular accumulation in hippocampus. Organelles like mitochondria, intracellular membranes, and endoplasmic reticulum have been identified as sites of A42 production and accumulation affecting cellular metabolism. However whether ozone exposure induces overproduction and/or accumulation of A42 in endoplasmic reticulum has not been studied. We evaluated this effect in the endoplasmic reticulum of hippocampal cells of rats exposed chronically to low doses of ozone $(0.25 \mathrm{ppm})$ at 7, 15, 30, 60, and 90 days. The effect of the presence of A42 in endoplasmic reticulum was analyzed evaluating the expression of the chaperone Syntaxin 5. Our results show an accumulation of A42 peptide in this organelle. It was observed by immunofluorescence and by WB in endoplasmic fractions from hippocampal cells of rats at 60 and 90 days of treatment. Significant overexpression of the chaperone Syntaxin 5 at 60 and 90 days of treatment was observed $\left({ }^{*} P<0.05\right)$. These results indicate that the exposure to environmental pollutants could be involved as a risk factor for neurodegenerative processes.
\end{abstract}

\section{Introduction}

Oxidative stress is produced as a consequence of pathological, dietary, and environmental factors. Many people living in large cities around the world are affected by environmental pollutants like ozone $\left(\mathrm{O}_{3}\right)$ and it has been demonstrated that chronic exposure to ozone $(0.025 \mathrm{ppm})$ similar to that reported in a day of high pollution in Mexico City causes a state of oxidative stress [1]. Oxidative stress is a major risk factor for Alzheimer's disease (AD). Besides, it has been shown that free radicals enhance the amyloid pathology of $\mathrm{AD}$ [2].

Several previous studies from our laboratory have shown that the chronic oxidative stress caused by $\mathrm{O}_{3}$ produces progressive neurodegeneration in rat hippocampi. In addition memory deterioration, motor activity deficits, lipid peroxidation, and mitochondrial dysfunction were also observed in the hippocampi [3-7].
More recently we have shown a direct relationship of $\beta A 42$ overproduction and intracellular accumulation with oxidative stress $[6,8]$.

Many reports have determined that the extracellular and intracellular accumulation of $\beta \mathrm{A} 42$ is involved in the development and progression of $\mathrm{AD}$ [8]. However, the precise mechanism of $\beta \mathrm{A} 42$ neurotoxicity is not completely understood.

It has been demonstrated that mitochondria, ER, and Golgi apparatus are targets of $\beta \mathrm{A} 42$, while ER dysfunction in $\mathrm{AD}$ is well documented [2,6,8-12]. However, whether oxidative damage precedes and contributes directly to the ER accumulation of the $\beta \mathrm{A} 42$ peptides remains unclear.

Previous studies have shown that $\beta \mathrm{A} 42$ can bind to different proteins and extracellular and intracellular macromolecules that affect normal neuronal function and it has been proposed that ER dysfunction is a distinctive hallmark 
in $\mathrm{AD}$. For example, the presence of $\beta \mathrm{A} 42$ in ER causes ER stress which in turn activates indicators of the unfolded protein response (UPR), a response activated by ER stress. This kind of alterations affects cytoskeleton integrity and leads to cell death by $\beta$ A42 presence in neurons [13]. Besides, the accumulation of $\beta \mathrm{A} 42$ induces apoptosis by activating different pathways as the crosstalk between ER and mitochondria. On the other hand mitochondrial dysfunction triggers caspases 3, 8, and 12 activation in cybrid cells, indicating that the death receptor and the ER-specific apoptotic pathways are also activated upon exposure to $\beta$ A42 [14-16].

It is well known that the mitochondrial pathway is one of the principal generators of oxidative stress. Nevertheless, the stress in the ER is involved or may be induced by oxidative stress in AD. For example, disturbances of calcium and ER homeostasis induce an oxidative stress state and potential $\beta$ A42 aggregation mediated by iron [17].

Our aim in this work was to demonstrate that the chronic exposure of rats to an environmental pollutant like $\mathrm{O}_{3}$ $(0.25 \mathrm{ppm})$ may cause an imbalance in the production of $\beta A 42$. Such imbalance may lead to the progressive accumulation of this peptide in the ER, inducing changes in the normal metabolism of the cells.

\section{Materials and Methods}

2.1. Animal and Animal Care. 72 male Wistar rats weighing 250-300 g were individually housed in acrylic boxes within a clean air box, and food was provided ad libitum (NutriCubo, Purina, USA). The control and treated rats were maintained in a temperature-controlled and humidity-controlled environmental bioterium. The animals were maintained and treated in accordance with the Norma Official Mexicana NOM-036-SSA 2-2002 and the Bioethics Committee of the Faculty of Medicine at the National Autonomous University of Mexico.

2.2. General Procedure. The rats were randomly separated into six experimental groups ( $n=12$ per group). Group 1 was exposed daily to a clear airstream free of $\mathrm{O}_{3}$ for $4 \mathrm{~h}$, and groups 2, 3, 4, 5, and 6 were exposed to $\mathrm{O}_{3}$ for $7,15,30,60$, and 90 days, respectively. The experimental groups were exposed to $0.25 \mathrm{ppm}$ of $\mathrm{O}_{3}$ for $4 \mathrm{~h}$ daily. One of these subgroups was used for immunohistochemical analyses, and the other group was used for cellular fractionation.

2.3. $\mathrm{O}_{3}$ Exposure. In order to expose them to chronic doses of ozone $(0.025 \mathrm{ppm})$ the animals were placed inside a chamber and the procedure was carried out essentially as described [7].

Two hours after the final exposure to clean air or $\mathrm{O}_{3}$, the animals from each group were anesthetized with sodium pentobarbital (50 mg/kg i.p.; Sedalpharma, Edo. de México, Mexico) and then decapitated.

The hippocampi of six animals from each group were obtained for Western Blot (WB), and the other three animals were transcardially perfused with $4 \%$ paraformaldehyde (Sigma-Aldrich Chemie, Germany) in $0.1 \mathrm{M}$ phosphate buffer (J.T. Baker, NJ; PB, Tecsiquim; pH 7.4) for the immunohistochemistry assays. The postfixation of the brains was made essentially as described previously [1]. Five-micrometer sagittal slices of the brain containing the hippocampus were obtained using a microtome (American Optical), mounted on slides, and stored.

2.4. Subcellular Fractionation. To isolate the endoplasmic reticulum fractions, the hippocampal cells were lysed with a Dounce homogenizer. Microsomes from these cells were isolated using the Endoplasmic Reticulum Isolation Kit (SigmaAldrich) according to the manufacturer's instructions. The ER fractions were used immediately for WB assays as described by Hernández-Zimbrón and Rivas-Arancibia [7].

2.5. Western Blot (WB). The production levels of $\beta A 42$ in the $\mathrm{RE}$ fractions were analyzed by gel electrophoresis and WB. The tissue was homogenized, and $50 \mu \mathrm{g}$ of protein from each sample was boiled and separated on a $4-12 \%$ SDS polyacrylamide gel (Invitrogen) for $45 \mathrm{~min}$ at 90 volts (V). The proteins were electrophoretically transferred onto PVDF membranes (Sigma-Aldrich, San Luis, MO, USA). The membranes were blocked with $4 \%$ fat-free milk in Tris buffer solution (TBS-T) with $0.01 \%$ Tween 20 (TBS-T) (Sigma-Aldrich, San Luis, MO, USA) overnight at $37^{\circ} \mathrm{C}$. After being blocked, the membranes were incubated individually with the following antibodies: rabbit anti- $\beta$ A42 (Abcam Inc., Boston, MA, USA) (1:2000). The anti-GRP78 antibody (ER marker) was used as loading control. To evaluate the effect of the presence of $\beta \mathrm{A} 42$ in the ER, the expression levels of Syntaxin 5 (Syx5) were evaluated and the antibody goat anti-Syntaxin 5 (Santa Cruz Biotechnology, CA, USA) was used $(1: 200)$.

The membranes were incubated overnight with gentle shaking at $4^{\circ} \mathrm{C}$ (Brinkmann OrbMix 110, Brinkmann, Germany). The membranes were rinsed with TBS-T and subsequently incubated for $2 \mathrm{~h}$ at room temperature (RT) in TBS-T containing goat anti-rabbit IgG conjugated to the horseradish peroxidase secondary antibody and diluted to $1: 1000$ (Santa Cruz Biotechnology, CA, USA) for $1 \mathrm{~h}$. The immunoreactive bands were detected by chemiluminescence (ECL; General Electric, Santa Clara, CA, USA). The intensity of each band was quantified using an imaging densitometer (model GS700). The intensities were analyzed using the open access ImageJ software (NIH) as we previously reported.

2.6. Double Immunofluorescence for $\beta A 42$. Rabbit monoclonal anti- $\beta$ A42 antibody (obtained from Abcam, MA, USA), Goat polyclonal anti-GRP78 (Santa Cruz Biotechnology, CA, USA), and goat polyclonal anti-Syx5 (Santa Cruz Biotechnology, CA, USA) were used for double IF to detect the $\beta$ A42 peptide in the ER of hippocampal cells of the rats.

Sagittal sections of each brain containing the hippocampus were paraffin-embedded, treated with a paraffin-removal and heat-retrieval solution (Biocare Medical), and placed in a Decloaking Chamber (Biocare Medical) for $5 \mathrm{~min}$. Then, the sections were rinsed with distilled water and treated with a blocking reagent (PBS-Bovine Serum Albumin (0.04\%)) for 1 hour, washed with $0.1 \mathrm{M}$ phosphate saline buffer, and incubated individually for $12 \mathrm{~h}$ at $4^{\circ} \mathrm{C}$ with anti- $\beta \mathrm{A} 42$ (dilution $1: 200)$. 

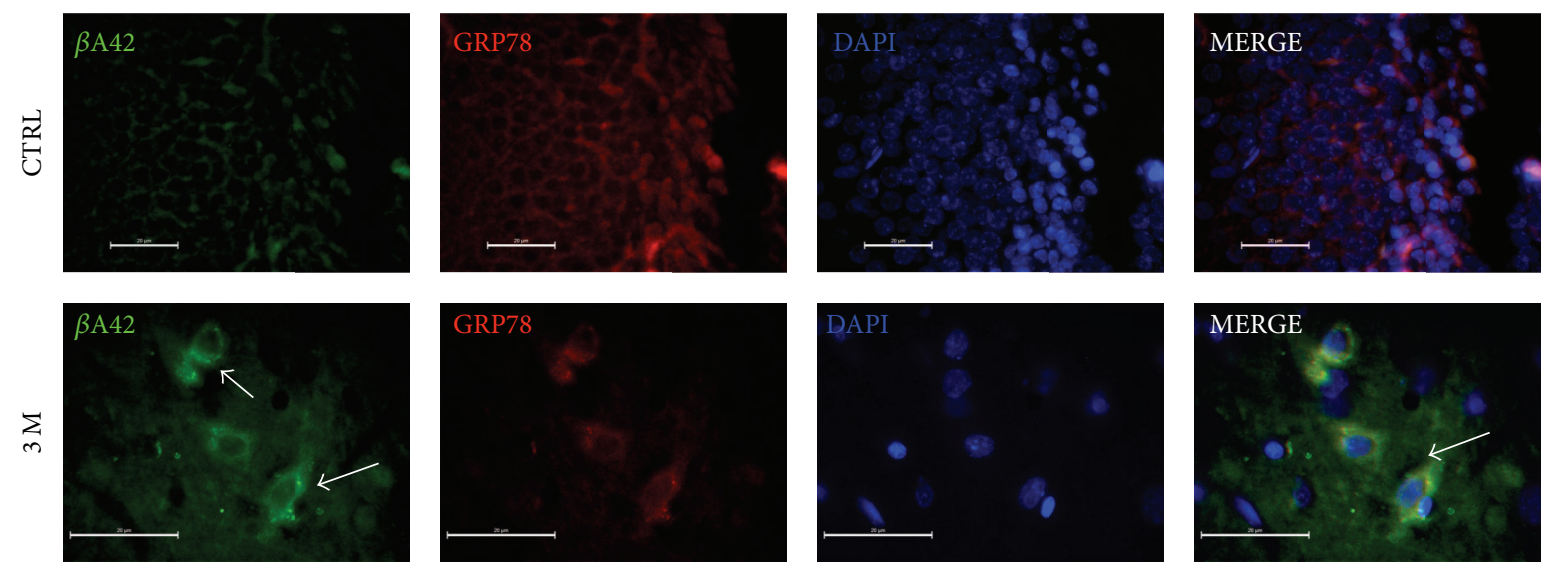

FIGURE 1: Triple IF to detect beta-amyloid 1-42 in the ER. Five micrometer-thick brain tissue sections form the hippocampi of the control group (CTRL) and the rats exposed to $\mathrm{O}_{3}(0.025 \mathrm{ppm})$ for 3 months $(3 \mathrm{M})$ are shown. Goat anti-GRP78 was used as ER marker. Rabbit anti$\beta A 42$ is shown in green and GRP78 in red. DAPI staining and merge between the red, green, and blue channels are shown (MERGE). The arrows indicate the intracellular $\beta$ A42 deposition and colocalization in ER. There was no $\beta$ A42 detection at 7, 15, 30, and 60 days of treatment (data not shown).

For the double immunofluorescence assays (IF), all primary antibodies were incubated overnight at $4^{\circ} \mathrm{C}$ and then rinsed three times with PBS/Triton (0.03\%) and incubated for 2 hours with secondary antibodies. Rabbit monoclonal anti- $\beta$ A42 and goat polyclonal anti-GRP78 (dilution 1:200) were used and visualized with Alexa Fluor 488 goat antirabbit IgG $(\mathrm{H}+\mathrm{L})$ and Alexa Fluor 594 donkey anti-goat $\operatorname{IgG}(\mathrm{H}+\mathrm{L})$, respectively. Rabbit monoclonal anti- $\beta \mathrm{A} 42$ and goat polyclonal anti-Syx5 (dilution 1:200) were used and visualized with Alexa Fluor 594 goat anti-rabbit IgG $(\mathrm{H}+\mathrm{L})$ and Alexa Fluor 488 mouse anti-goat $\operatorname{IgG}(\mathrm{H}+\mathrm{L})$. All the secondary antibodies were from Molecular Probes, OR, USA. Finally, the slides were rinsed and mounted onto glass slides in Vectashield medium (Vector Laboratories, Burlingame, CA, USA) containing 4',6-diamino-2-phenylindole (DAPI). Representative brain sections from each group were processed in parallel afterwards and these sections were observed through a Leica DM-LS epifluorescence microscope at 40x and 100x (Leica Microsystems, Wetzlar, GmbH, Germany). The fluorochromes were visualized with their specific filters and analyzed in three channels.

2.7. Statistical Analysis. All of the data are expressed as mean \pm SEM. ANOVA analyses followed by Fisher's LSD post hoc, Bonferroni's, and Tukey's tests were used for multiple comparisons. Prism Graph Pad Software was used (Systat Software, Inc., Point Richmond, CA, USA) and differences were considered significant at $P<0.05$.

\section{Results}

3.1. BA42 Accumulation in RE Fraction under Oxidative Stress Conditions. We have reported that oxidative stress state caused by $\mathrm{O}_{3}$ exposure induces intracellular $\beta$ A42 accumulation as well as mitochondrial accumulation in our neurodegeneration model $[6,7]$. To prove the accumulation of this peptide in ER of hippocampal cells of rats treated with low doses of $\mathrm{O}_{3}$, we performed double IF assays in brain sections derived from both control and experimental groups. The double IF assays showed qualitative increases in the intrareticular accumulation of $\beta \mathrm{A} 42$ in the hippocampal dentate gyrus cells only at 90 days ( $3 \mathrm{M}$ ) of treatment (Figure 1).

\subsection{Accumulation of $\beta A 42$ in ER Isolated Fractions Caused} by $\mathrm{O}_{3}$ Exposure. Next, in order to demonstrate the accumulation of $\beta A 42$ in the ER fractions, the level of $\beta A 42$ was quantified by $\mathrm{WB}$ and densitometry analyses from the rat hippocampal cells isolated on days 0 (control), 7, 15, 30, 60, and 90 of exposure to $\mathrm{O}_{3}$. The most representative values of $\beta A 42$ were obtained. As we can see in Figure $2(\mathrm{a}), \sim 3.5 \mathrm{kDa}$ band for the $\beta$ A 42 monomer was detected only at 90 days of ozone treatment, demonstrating $\beta$ A 42 accumulation in the isolated ER fractions when compared with the control group. The densitometry analysis showed a significant increase of $\beta \mathrm{A} 42$ accumulation in ER fractions (Figure 2(b)).

3.3. Oxidative Stress Causes Overexpression of Syx 5 in ER. To demonstrate the possible effect of oxidative stress and betaamyloid accumulation in ER metabolism, we have performed $\mathrm{WB}$ and IF assays to detect changes in the expression levels of Syx 5 protein. Syx 5 protein has been related with communication processes between mitochondria and ER and described as a chaperone as well. Our WB results showed that ozone exposure alters the expression of Syx 5 at 60 and 90 days (Figures 2(c) and 2(d)). Besides, the overexpression of Syx 5 was observed by IF (Figure 3(a)) at 90 days and by immunohistochemistry principally at 60 and 90 days of treatment (Figure 3(b)).

\section{Discussion}

In this study we have demonstrated that $\mathrm{O}_{3}$ exposure $(0.25 \mathrm{ppm})$ induced a significant increase of Syntaxin 5 and accumulation of $\beta$ A42 peptide in the ER in cells of the dentate 


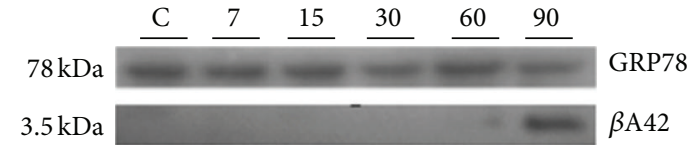

(a)

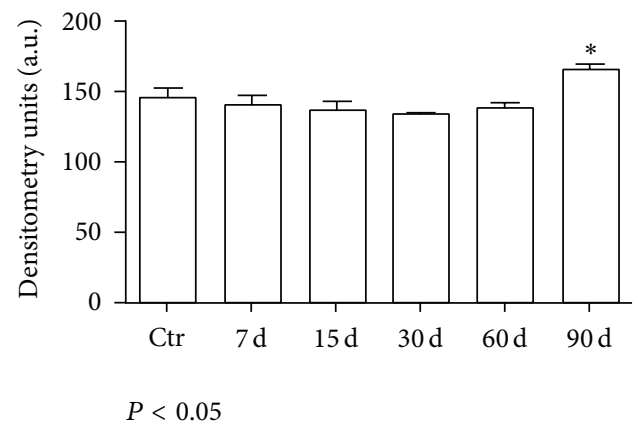

(b)

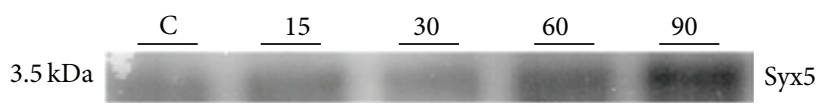

(c)

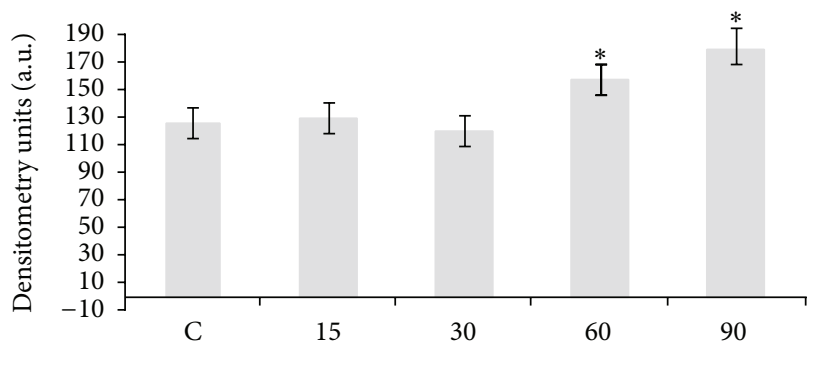

$P<0.05$

(d)

FIGURE 2: Immunodetection of $\beta \mathrm{A} 42$ and Syx 5 in the ER protein extracts of rat hippocampi following different $\mathrm{O}_{3}$ treatments. (a) WB for $\beta$ A42 in the ER fractions. The ER marker GRP78 was used as loading control and ER isolation control. (b) Densitometry analyses of the WB assays for $\beta$ A42. $\beta$ A42 production increased by the end of the ozone treatment. The differences became statistically significant at 90 days of $\mathrm{O}_{3}$ exposure for $\beta \mathrm{A} 42 ;{ }^{*} \mathrm{P}<0.05$. (c) WB for Syntaxin 5 from the ER fraction (control, 15, 30, 60, and 90 days). Rabbit anti-Syntaxin 5 was used for immunodetection and was visualized by chemiluminescence. (d) Densitometry analyses of the WB assays. There were significant changes in Syntaxin 5 expression at 60 and 90 days of treatment. The differences between the control and experimental groups were statistically significant; ${ }^{*} P<0.05$.
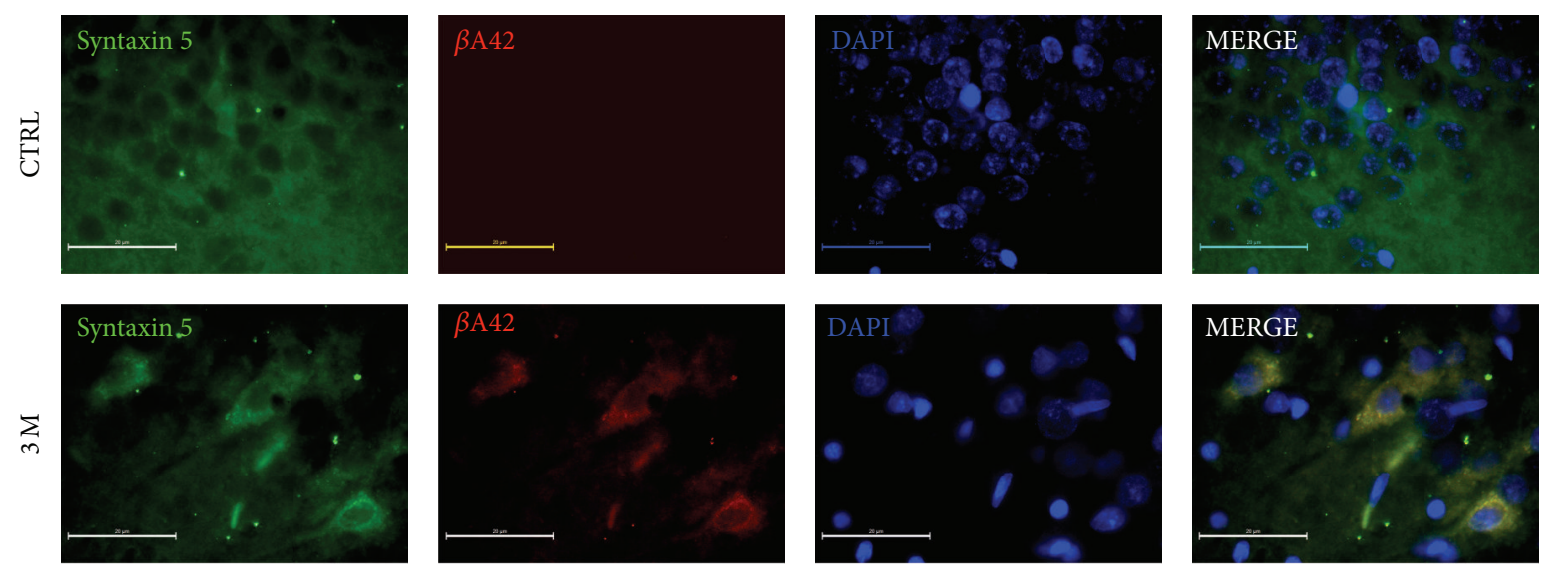

(a)
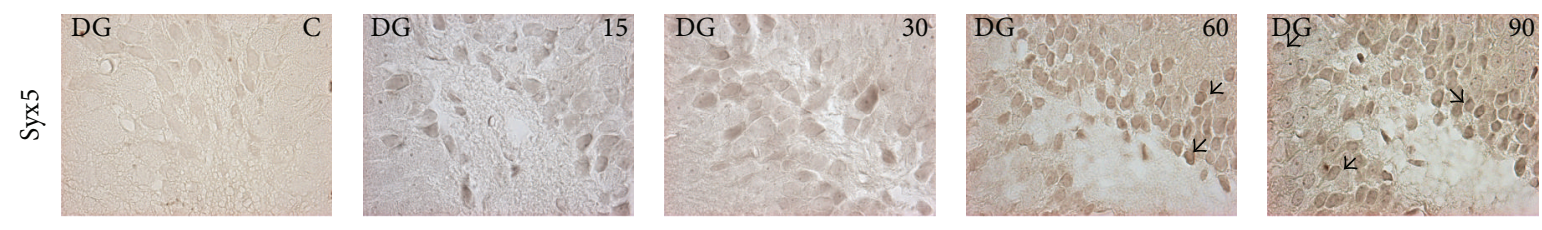

(b)

FIGURE 3: Immunodetection of Syx 5 and $\beta \mathrm{A} 42$ in the ER. Five micrometer-thick brain tissue sections form hippocampi of the control group $(C T R L)$ and the rats exposed to $\mathrm{O}_{3}(0.25 \mathrm{ppm})$ for 3 months $(3 \mathrm{M})$ are shown. Rabbit anti- $\beta$ A42 is shown in red and Syntaxin 5 in green. DAPI staining (DAPI) and merge between red, green, and blue channels are shown (MERGE). The arrows indicate the overexpression of Syx 5 and the intracellular $\beta$ A42 deposition and colocalization in the ER (a). There was no $\beta$ A42 detection at 7, 15, 30, and 60 days of treatment (data not shown). (b) Immunohistochemistry for Syx 5 in brain slides of control (C), 15, 30, 60, and 90 days of treatment showing the overexpression of Syx 5 under $\mathrm{O}_{3}$ treatment. 
gyrus. Changes in the expression patterns of the chaperone Syntaxin 5 precede the accumulation of $\beta \mathrm{A} 42$ after the treatment with this environmental pollutant.

$\mathrm{AD}$ is a neurodegenerative disease with a complex and progressive pathological phenotype [18]. The $\beta$ A42 peptide, a hallmark of $\mathrm{AD}$, is produced through the sequential cleavage of APP by $\beta$ - and $\gamma$-secretase [19]. Several reports have demonstrated that the origin and progression of $\mathrm{AD}$ are highly correlated with oxidative stress status. Oxidative damage, mitochondrial dysfunction, and age dependent increases of reactive oxygen species (ROS) have been identified as key factors in the development of sporadic AD [20-22]. Besides, oxidative stress may contribute to neuronal degeneration in $\mathrm{AD}$ by increasing iron and lipid peroxidation, protein oxidation, and some markers of oxidative stress that are present in the senile plaques in AD [23-25].

Previous results from our laboratory and other groups have demonstrated that the chronic administration of $\mathrm{O}_{3}$ $(0.25 \mathrm{ppm})$ induces an oxidative stress status, increased lipid peroxidation levels in different brain structures, and morphological and structural changes in neurons and elevates hippocampal superoxide accumulation [26-29].

More recently, we demonstrated that rats exposed to $\mathrm{O}_{3}$ overproduce $\beta \mathrm{A} 42$, inducing intramitochondrial $\beta \mathrm{A} 42$ accumulation [7]. Moreover, ER oxidative damage has also been well documented in AD. It is well known that the mitochondrial pathway is the main generator of ROS. ER stress is caused by ROS in $\mathrm{AD}$, while the disturbances of calcium and ER homeostasis induce an oxidative stress state which increases Syntaxin 5 and lead to potential $\beta$ A 42 aggregation. However, the precise mechanism that links ER oxidative damage with abnormal $\beta$ A 42 overproduction and accumulation has not been elucidated [30].

Based on our previous results showing mitochondrial accumulation of $\beta$ A42 we performed assays to demonstrate the effect of ozone exposure in ER. We found an increase of Syntaxin 5 before the accumulation of $\beta \mathrm{A} 42$ in the ER of hippocampal cells. Both effects could be related to ER metabolism disturbance in our progressive neurodegeneration model.

First, we suggest that the overproduction or the increase in $\beta \mathrm{A} 42$ accumulation in hippocampal cells could be related with the increase of ROS production; second, it could be related with the accumulation and processing of APP in these cells. As we have demonstrated before, there is an increase of the amyloidogenic pathway activity in mitochondria [7]. This overactivity and overproduction of beta-amyloid peptide could be associated with the accumulation in ER membranes, mitochondrial membranes, or Mitochondrial Associated Membranes (MAM) as has been suggested by Pereira, 2013 [31-36].

As we have mentioned before, ER is a system formed by continuous membranes. It comprises different architectural shapes of the nuclear envelope, sheet-like structures, containing polyribosomes and smooth tubules present throughout the cell. This organelle is involved in the synthesis, folding, structural maturation, control, and trafficking of integral membrane and secreted proteins in cells. According to its membranous structure, there is evidence demonstrating that MAM are physiological interactions between the ER and mitochondria and have different functions such as lipid transport and synthesis, ER calcium regulation, mitochondrial calcium release, mitochondrial movement and morphology, and protein trafficking.

It has been recently described that PS2 is a protein present in MAM and that the complete $\gamma$-secretase complex is present in both ER and MAM [32, 33, 37-40].

In accordance with our results that show the accumulation of $\beta \mathrm{A} 42$ in $\mathrm{ER}$, we could suggest that the $\beta \mathrm{A} 42$ overproduced and PS1 overexpressed in mitochondria may be transported to ER through MAM. The overcleavage of APP by PS1 and PS2 in MAM has been reported in AD and under oxidative stress conditions, as it has been deeply reviewed by $[37,40]$. It should be pointed out that the connectivity is increased between ER and mitochondria in $\mathrm{AD}$, and it might be happening in the model present in this work [7, 32, 33]. Another feasible mechanism to explain the $\beta$ A 42 presence in ER is the oxidative stress caused by $\mathrm{O}_{3}$ exposure per se.

The increase in the production and accumulation of betaamyloid peptide in hippocampal cells may be correlated with the significant increase in PS1 expression and a reduced expression of ADAM9 under oxidative stress conditions. This indicates that low doses of $\mathrm{O}_{3}$ elicited the overactivation of the amyloidogenic pathway [7].

Then, to demonstrate the effect of the accumulation of $\beta$ A42 in ER, we evaluate Syntaxin 5, a chaperone directly related with the communication between ER and mitochondria. For example, Syntaxin 5 and Syntaxin 17 facilitate the transport of cholesterol to mitochondria $[33,34]$.

Syntaxins are a family of vesicular transport receptors involved in membrane traffic through both the constitutive and regulated secretory pathways [41]. One of Syntaxin 5 functions is to shape the ER and is expressed ubiquitously in many cell types and has been located in the ER and Golgi compartment of the early secretory pathways [42]. Besides, Syntaxin 5 is thought to regulate the potential targeting and fusion carrier vesicles at multiple membrane fusion interfaces by affecting the selective combination of the SNARE complex with other SNARE-related proteins (6-9). Some reports have demonstrated that Syntaxin 5 specifically interacts with PS holoproteins, but not with the N-terminal or C-terminal fragments of PS, and the overexpression was shown to upregulate $\beta$ APP accumulation in the ER through the Golgi compartments, attenuate accumulation of the C-terminal fragment of $\beta A P P$, and reduce $A \beta$ secretion [16].

Our results show that $\mathrm{O}_{3}$ exposure induces the overexpression of the chaperone Syntaxin 5 at 60 and 90 days of treatment observed by WB (Figures $2(\mathrm{c})$ and $2(\mathrm{~d})$ ) and by IF and IMHQ (Figure 3).

Besides, it has been described that Syntaxin 5 interacts with PSs in ER and the Golgi compartment and its overexpression causes the accumulation of the PS and $\beta$ APP as well as the reduction in the level of $\mathrm{A} \beta$ secretion $[40,42]$. Then we could suggest that the accumulation of $A \beta$ in ER could be in part a consequence of the overexpression of Syntaxin 5 (Figure 4). 

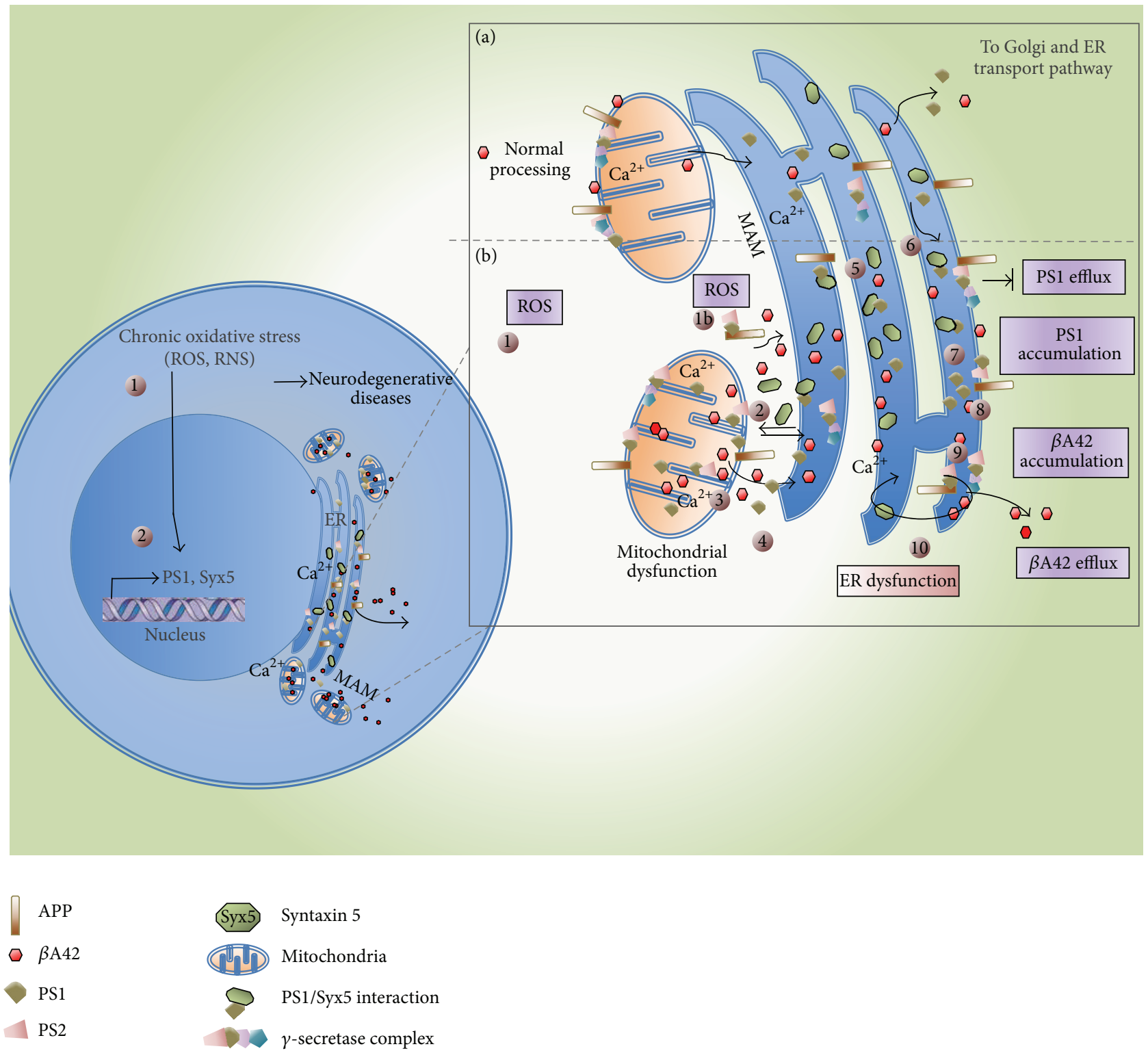

FIGURE 4: Schematic representation of the suggested mechanisms related with $\beta$ A42 accumulation in ER. (a) Normal metabolism of mitochondria and ER under no oxidant stress conditions. Normal processing of $\beta$ A42. (b) Metabolism alterations under oxidant stress conditions. (1) $\mathrm{O}_{3}$ induces an oxidative stress state causing PS1 and Syx5 overexpression. (1b) ROS induces by itself the overcleavage of APP by PS1 and PS2. (2) PS1 and Syx5 accumulate in mitochondria, MAM, and ER. (3) $\beta A 42$ accumulation in mitochondria, MAM, and ER. (4) Transport of PS1, Syx5, and $\beta$ A42 through MAM. (5) Syx5 excess in ER. (6) Interaction Syx5/PS1 blocks the PS1 normal efflux. (7) PS1 accumulation. (8) PS1 transport to $\gamma$-secretase complex. (9) Accumulation of $\beta$ A42 in ER. (10) ER dysfunction.

As we mentioned before, PSs are located in the ER and although they lack proteolytic processing they are active molecules in AD with $\gamma$-secretase activity.

The increase of the expression of PS1 in the late compartment of the secretory pathways may lead to higher levels of $\beta A 40$ and $\beta A 42$ production [43]. We have reported that there is an overexpression of PS1 in the mitochondria of hippocampal cells at 60 and 90 days of $\mathrm{O}_{3}$ exposure [7]. We suggest that PS1 overexpressed may be transported to ER through MAM or a different mechanism. Then, PS1 and PS2 in the ER could bind to Syntaxin 5, inhibiting the degradation or transport through the ER associated pathway. In addition, it has been demonstrated that Syntaxin 5 in cooperation with PS alters the production of $\beta$ A peptides. It affects the processing and/or trafficking of the fragment called $\beta$ APP, which arrives at the site where $\gamma$-secretase appears.

Additionally, it has been demonstrated that Syntaxin 5 overexpression reduced the concentration of $\mathrm{A} \beta 40$ peptide secreted, a fact that supports our results and suggestions $[44,45]$.

Through the methodological approach that we have used in this work we cannot confirm that there are defects in 
the ER-Golgi transport; only we suggest this point based on the bibliographic review. For example, some authors suggest that it may be possible that perturbation of Syx 5 isoform expression in neuronal cells cause some changes in $\mathrm{A} \beta$ production in the central nervous system. Suga et al. have demonstrated that Syx 5 binds to the PS holoprotein in the ER and Golgi compartments of neuronal cells and they modulate the metabolism and trafficking of $\beta$ APP. Because PS and $\beta$ APP are sorted and processed along the secretory and endocytotic pathways, alterations in the transport machinery could affect the trafficking of these proteins, affecting the generation and secretion of $\mathrm{A} \beta$. Thus, malfunction of this chaperone may cause the accumulation of excess $\mathrm{A} \beta$ peptides found in late-onset AD.

Finally, the ER stress and oxidative stress cause changes in the pattern of APP processing in affected neurons, increasing the amount of $\beta \mathrm{A} 42$ peptide. ROS also play a role in cell signaling through a mechanism known as redox signaling. Any alteration in the cellular antioxidant defense system and the increase in ROS lead to redox signaling alterations, which are involved in neurodegenerative processes and directly in the overproduction of $\beta$ A42 $[5-7,46]$.

$\mathrm{A} \beta$ and oxidative stress together are able to trigger an ER stress response, leading to synaptic and neuronal loss, increasing the levels of markers of the ER stress response (UPR), and activating caspases pathway and apoptosis in cortical neurons $[13,15,16,36,47]$. Besides, the presence of $\mathrm{A} \beta$ in the ER induces the expression of some chaperones like GRP78 and GRP94, involved in the caspase-12 activation and cell death [48].

\section{Conclusion}

Cell injury due to ER stress has emerged as a key contributor to the pathophysiology of a wide range of neurodegenerative human diseases like Alzheimer's disease and pathological aging. Additional studies are needed to further investigate the impact of $\beta$ A42 accumulation in the ER. Nevertheless, the present results help to show that the current levels of environmental pollutants in highly polluted cities might induce and participate in the development of neurodegenerative processes by oxidative stress.

\section{Competing Interests}

The authors report no conflict of interests.

\section{Acknowledgments}

This work was supported by DGAPA-IN221114 and CONACyT-219703 to Selva Rivas-Arancibia.

\section{References}

[1] S. Rivas-Arancibia, R. Guevara-Guzmán, Y. López-Vidal et al., "Oxidative stress caused by ozone exposure induces loss of brain repair in the hippocampus of adult rats," Toxicological Sciences, vol. 113, no. 1, pp. 187-197, 2009.
[2] H. Misonou, M. Morishima-Kawashima, and Y. Ihara, "Oxidative stress induces intracellular accumulation of amyloid $\beta$ protein $(\mathrm{A} \beta)$ in human neuroblastoma cells," Biochemistry, vol. 39, no. 23, pp. 6951-6959, 2000.

[3] M. R. Avila-Costa, L. Colín-Barenque, T. I. Fortoul et al., "Memory deterioration in an oxidative stress model and its correlation with cytological changes on rat hippocampus CA1," Neuroscience Letters, vol. 270, no. 2, pp. 107-109, 1999.

[4] M. R. Avila-Costa, L. Colín-Barenque, T. I. Fortoul et al., "Motor impairments in an oxidative stress model and its correlation with cytological changes on rat striatum and prefrontal cortex," International Journal of Neuroscience, vol. 108, no. 3-4, pp. 193200, 2001.

[5] C. Dorado-Martínez, C. Paredes-Carbajal, D. Mascher, G. Borgonio-Pérez, and S. Rivas-Arancibia, "Effects of different ozone doses on memory, motor activity and lipid peroxidation levels, in rats," International Journal of Neuroscience, vol. 108, no. 3-4, pp. 149-161, 2001.

[6] S. Rivas-Arancibia, C. Gallegos-Ríos, N. Gomez-Crisostomo et al., "Oxidative stress and neurodegenerative disease," in Neurodegenerative Diseases-Processes, Prevention, Protection and Monitoring, R. C.-C. Chang, Ed., chapter 3, InTech, Rijeka, Croatia, 2011.

[7] L. F. Hernández-Zimbrón and S. Rivas-Arancibia, "Oxidative stress caused by ozone exposure induces $\beta$-amyloid 1-42 overproduction and mitochondrial accumulation by activating the amyloidogenic pathway," Neuroscience, vol. 304, pp. 340-348, 2015.

[8] L. F. Hernández-Zimbrón and S. Rivas-Arancibia, "Deciphering an interplay of proteins associated with amyloid $\beta 1-42$ peptide and molecular mechanisms of Alzheimer's disease," Reviews in the Neurosciences, vol. 25, no. 6, pp. 773-783, 2014.

[9] M. Manczak, T. S. Anekonda, E. Henson, B. S. Park, J. Quinn, and P. H. Reddy, "Mitochondria are a direct site of $\mathrm{A} \beta$ accumulation in Alzheimer's disease neurons: implications for free radical generation and oxidative damage in disease progression," Human Molecular Genetics, vol. 15, no. 9, pp. 1437$1449,2006$.

[10] M. P. Horowitz and J. T. Greenamyre, "Mitochondrial iron metabolism and its role in neurodegeneration," Journal of Alzheimer's Disease, vol. 20, no. 2, pp. S551-S568, 2010.

[11] P. I. Moreira, C. Carvalho, X. Zhu, M. A. Smith, and G. Perry, "Mitochondrial dysfunction is a trigger of Alzheimer's disease pathophysiology," Biochimica et Biophysica Acta-Molecular Basis of Disease, vol. 1802, no. 1, pp. 2-10, 2010.

[12] G. Joshi, Y. Chi, Z. Huang, and Y. Wang, "A $\beta$-induced Golgi fragmentation in Alzheimer's disease enhances A $\beta$ production," Proceedings of the National Academy of Sciences of the United States of America, vol. 111, no. 13, pp. E1230-E1239, 2014.

[13] K. I. Seyb, S. Ansar, J. Bean, and M. L. Michaelis, “ $\beta$-amyloid and endoplasmic reticulum stress reponses in primary neurons: effects of drugs that interact with the cytoskeleton," Journal of Molecular Neuroscience, vol. 28, no. 2, pp. 111-124, 2006.

[14] Z. F. Yu, H. Luo, W. Fu, and M. P. Mattson, "The endoplasmic reticulum stress-responsive protein GRP78 protects neurons against excitotoxicity and apoptosis: suppression of oxidative stress and stabilization of calcium homeostasis," Experimental Neurology, vol. 155, no. 2, pp. 302-314, 1999.

[15] T. Nakagawa, H. Zhu, N. Morishima et al., "Caspase-12 mediates endoplasmic-reticulum-specific apoptosis and cytotoxicity by amyloid- $\beta$," Nature, vol. 403, no. 6765, pp. 98-103, 2000. 
[16] R. O. Costa, E. Ferreiro, I. Martins et al., "Amyloid $\beta$-induced ER stress is enhanced under mitochondrial dysfunction conditions," Neurobiology of Aging, vol. 33, no. 4, pp. 824.e5-824.e16, 2012.

[17] L. Chami and F. Checler, "BACE1 is at the crossroad of a toxic vicious cycle involving cellular stress and $\beta$-amyloid production in Alzheimer's disease," Molecular Neurodegeneration, vol. 7, article 52, 2012.

[18] J. Yao, S. Chen, Z. Mao, E. Cadenas, and R. D. Brinton, "2-deoxy$\mathrm{D}$-glucose treatment induces ketogenesis, sustains mitochondrial function, and reduces pathology in female mouse model of Alzheimer's disease," PLoS ONE, vol. 6, no. 7, Article ID e21788, 2011.

[19] F. M. LaFerla, K. N. Green, and S. Oddo, "Intracellular amyloid$\beta$ in Alzheimer's disease," Nature Reviews Neuroscience, vol. 8, no. 7, pp. 499-509, 2007.

[20] P. H. Reddy and M. F. Beal, "Are mitochondria critical in the pathogenesis of Alzheimer's disease?" Brain Research Reviews, vol. 49, no. 3, pp. 618-632, 2005.

[21] R. H. Swerdlow and S. M. Khan, "A "mitochondrial cascade hypothesis" for sporadic Alzheimer's disease," Medical Hypotheses, vol. 63, no. 1, pp. 8-20, 2004.

[22] P. H. Reddy, M. Manczak, P. Mao, M. J. Calkins, A. P. Reddy, and U. Shirendeb, "Amyloid- $\beta$ and mitochondria in aging and Alzheimer's disease: Implications for synaptic damage and cognitive decline," Journal of Alzheimer's Disease, vol. 20, no. 2, pp. S499-S512, 2010.

[23] I. Kruman, A. J. Bruce-Keller, D. Bredesen, G. Waeg, and M. P. Mattson, "Evidence that 4-hydroxynonenal mediates oxidative stress-induced neuronal apoptosis," The Journal of Neuroscience, vol. 17, no. 13, pp. 5089-5100, 1997.

[24] R. J. Mark, Z. Pang, J. W. Geddes, K. Uchida, and M. P. Mattson, "Amyloid $\beta$-peptide impairs glucose transport in hippocampal and cortical neurons: Involvement of membrane lipid peroxidation," Journal of Neuroscience, vol. 17, no. 3, pp. 1046-1054, 1997.

[25] M. Padurariu, A. Ciobica, R. Lefter, I. L. Serban, C. Stefanescu, and R. Chirita, "The oxidative stress hypothesis in Alzheimer's disease," Psychiatria Danubina, vol. 25, no. 4, pp. 401-409, 2013.

[26] S. Rivas-Arancibia, C. Dorado-Martínez, L. Colin-Barenque, K. M. Kendrick, C. De La Riva, and R. Guevara-Guzmán, "Effect of acute ozone exposure on locomotor behavior and striatal function," Pharmacology Biochemistry and Behavior, vol. 74, no. 4, pp. 891-900, 2003.

[27] S. Rivas-Arancibia, L. F. Hernández-Zimbrón, E. RodríguezMartínez, G. Borgonio-Pérez, V. Velumani, and J. DuránBedolla, "Chronic exposure to low doses of ozone produces a state of oxidative stress and blood-brain barrier damage in the hippocampus of rat," Advances in Bioscience and Biotechnology, vol. 4, no. 11, pp. 24-29, 2013.

[28] M. Angoa-Pérez, H. Jiang, A. I. Rodríguez, C. Lemini, R. A. Levine, and S. Rivas-Arancibia, "Estrogen counteracts ozoneinduced oxidative stress and nigral neuronal death," NeuroReport, vol. 17, no. 6, pp. 629-633, 2006.

[29] M. L. Mokoena, B. H. Harvey, D. W. Oliver, and C. B. Brink, "Ozone modulates the effects of imipramine on immobility in the forced swim test, and nonspecific parameters of hippocampal oxidative stress in the rat," Metabolic Brain Disease, vol. 25, no. 2, pp. 125-133, 2010.

[30] O. Ghribi, "The role of the endoplasmic reticulum in the accumulation of $\beta$-amyloid peptide in Alzheimer's disease," Current Molecular Medicine, vol. 6, no. 1, pp. 119-133, 2006.
[31] C. M. F. Pereira, "Crosstalk between endoplasmic reticulum stress and protein misfolding in neurodegenerative diseases," ISRN Cell Biology, vol. 2013, Article ID 256404, 22 pages, 2013.

[32] E. Area-Gomez, A. J. C. De Groof, I. Boldogh et al., "Presenilins are enriched in endoplasmic reticulum membranesassociated with mitochondria," American Journal of Pathology, vol. 175, no. 5, pp. 1810-1816, 2009.

[33] Y. Lin, X. Hou, W. Shen et al., "SNARE-mediated cholesterol movement to mitochondria supports steroidogenesis in rodent cells," Molecular Endocrinology, vol. 30, no. 2, pp. 234-247, 2016.

[34] M. Hamasaki, N. Furuta, A. Matsuda et al., "Autophagosomes form at ER-mitochondria contact sites," Nature, vol. 495, no. 7441, pp. 389-393, 2013.

[35] K. Nishitsuji, T. Tomiyama, K. Ishibashi et al., "The E693 mutation in amyloid precursor protein increases intracellular accumulation of amyloid $\beta$ oligomers and causes endoplasmic reticulum stress-induced apoptosis in cultured cells," American Journal of Pathology, vol. 174, no. 3, pp. 957-969, 2009.

[36] T. Umeda, T. Tomiyama, N. Sakama et al., "Intraneuronal amyloid $\beta$ oligomers cause cell death via endoplasmic reticulum stress, endosomal/lysosomal leakage, and mitochondrial dysfunction in vivo," Journal of Neuroscience Research, vol. 89, no. 7, pp. 1031-1042, 2011.

[37] T. Simmen, E. M. Lynes, K. Gesson, and G. Thomas, "Oxidative protein folding in the endoplasmic reticulum: tight links to the mitochondria-associated membrane (MAM)," Biochimica et Biophysica Acta-Biomembranes, vol. 1798, no. 8, pp. 1465-1473, 2010.

[38] E. Zampese, C. Fasolato, T. Pozzan, and P. Pizzo, "Presenilin-2 modulation of ER-mitochondria interactions: FAD mutations, mechanisms and pathological consequences," Communicative \& Integrative Biology, vol. 4, no. 3, pp. 357-360, 2011.

[39] A. Raturi and T. Simmen, "Where the endoplasmic reticulum and the mitochondrion tie the knot: the mitochondriaassociated membrane (MAM)," Biochimica et Biophysica ActaMolecular Cell Research, vol. 1833, no. 1, pp. 213-224, 2013.

[40] E. A. Schon and E. Area-Gomez, "Mitochondria-associated ER membranes in Alzheimer disease," Molecular and Cellular Neuroscience, vol. 55, pp. 26-36, 2013.

[41] C. Hetz, E. Chevet, and S. A. Oakes, "Proteostasis control by the unfolded protein response," Nature Cell Biology, vol. 17, no. 7, pp. 829-838, 2015.

[42] K. Miyazaki, Y. Wakana, C. Noda, K. Arasaki, A. Furuno, and M. Tagaya, "Contribution of the long form of syntaxin 5 to the organization of the endoplasmic reticulum," Journal of Cell Science, vol. 125, no. 23, pp. 5658-5666, 2012.

[43] K. Suga, T. Tomiyama, H. Mori, and K. Akagawa, "Syntaxin 5 interacts with presenilin holoproteins, but not with their $\mathrm{N}$ - or C-terminal fragments, and affects $\beta$-amyloid peptide production," Biochemical Journal, vol. 381, no. 3, pp. 619-628, 2004.

[44] K. Suga, A. Saito, T. Tomiyama, H. Mori, and K. Akagawa, "The syntaxin 5 isoforms Syx5 and Syx5L have distinct effects on the processing of $\beta$-amyloid precursor protein," Journal of Biochemistry, vol. 146, no. 6, pp. 905-915, 2009.

[45] N. K. Jha, S. K. Jha, R. Kar, R. K. Ambasta, and P. Kumar, "Role of oxidative stress, ER stress and ubiquitin proteasome system in neurodegeneration," MOJ Cell Science and Report, vol. 1, no. 2, Article ID 00010, 2014.

[46] B. P. Yu, "Cellular defenses against damage from reactive oxygen species," Physiological Reviews, vol. 74, no. 1, pp. 139-162, 1994. 
[47] L. F. Hernández-Zimbrón, J. Luna-Muñoz, R. Mena et al., "Amyloid- $\beta$ peptide binds to cytochrome C oxidase subunit 1," PLoS ONE, vol. 7, no. 8, Article ID e42344, 2012.

[48] R. J. Viana, C. J. Steer, and C. M. Rodrigues, "Amyloid- $\beta$ peptide-induced secretion of endoplasmic reticulum chaperone glycoprotein GRP94," Journal of Alzheimer's Disease, vol. 27, no. 1, pp. 61-73, 2011. 

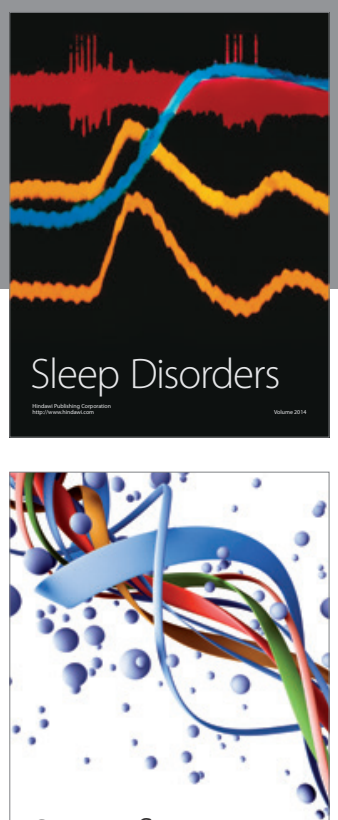

Scientifica
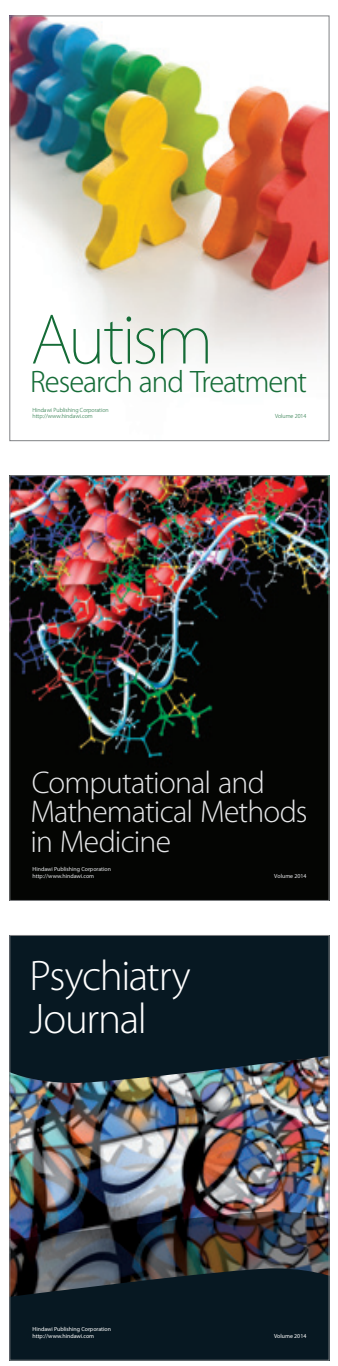
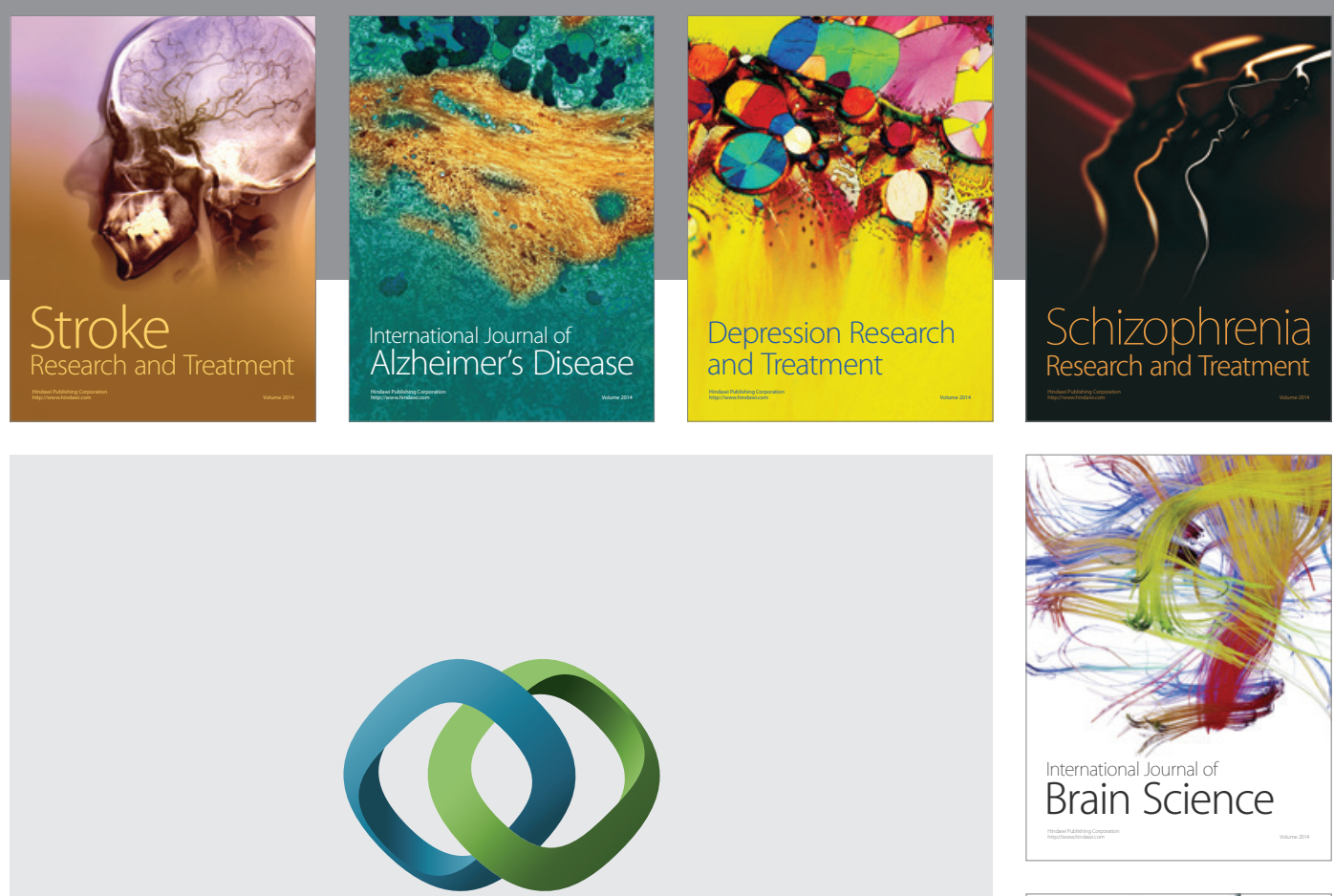

\section{Hindawi}

Submit your manuscripts at

http://www.hindawi.com
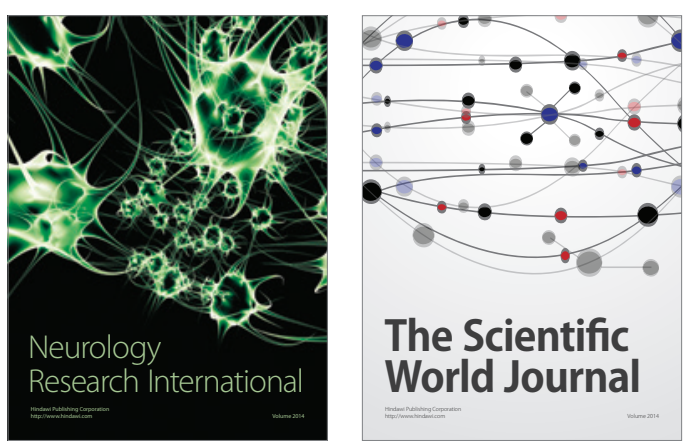

The Scientific World Journal

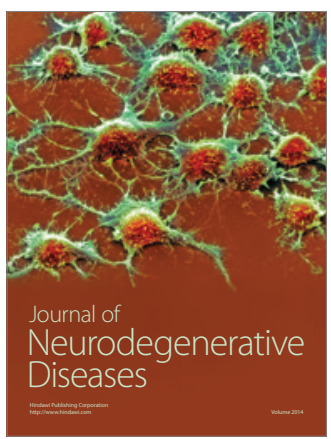

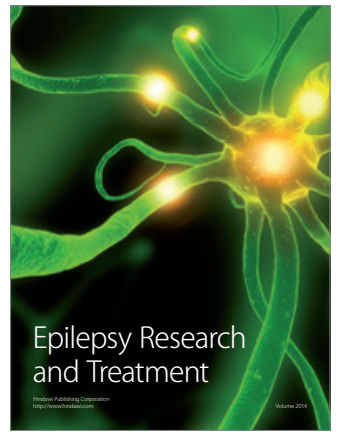

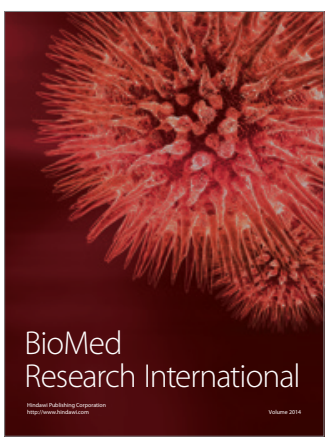

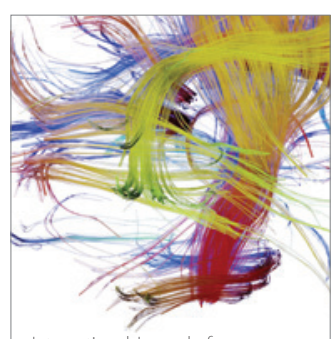

Brain Science

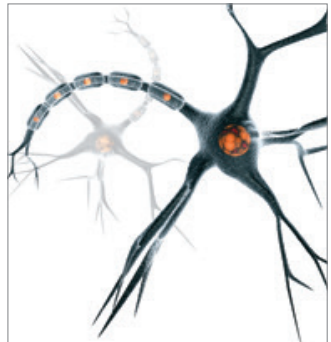

Neural Plasticity
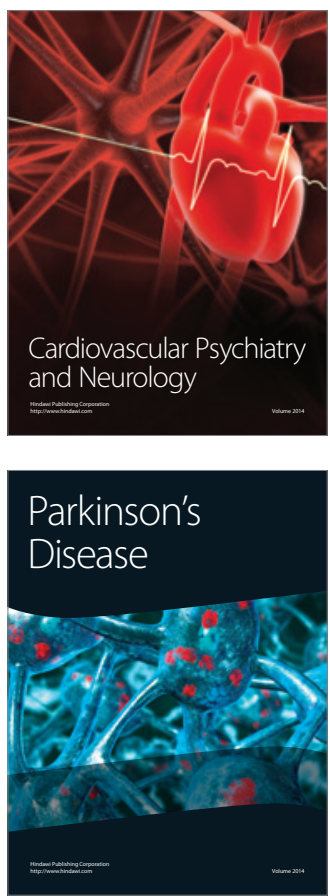